\title{
Métodos de evaluación de la calidad posicional en Hispanoamérica: análisis de la situación
}

\author{
F. Javier Ariza-López* \\ Emerson Xavier** \\ Elena Chicaiza** \\ Xavier Buenaño ${ }^{* * * *}$
}

Recibido el 15 de diciembre de 2016; aceptado el 22 de mayo de 2017

\begin{abstract}
Positional accuracy constitutes a primordial aspect of geographic information quality in any geomatics application and a key element to get interoperability. The aim of this publication is identify the methods of positional accuracy assessment that are using in Hispano-American countries and within them, some that worked in the project: "Diagnosis of current situation of methodologies and procedures applied in geographic information quality evaluation", funded by the Pan American Institute for Geography and History. The standards that are been applied are linked with American standards such as: EMAS, NMAS, NSSDA, PSADG. The most of these standards assume normality, but there are new trends such that opened by UNE 48002:2016, this Spanish standard opens a new framework. Finally, results of a survey about national maturity levels related with positional accuracy assessment conclude that positional-accuracy-assessment methods being applied in analyzed countries - are in an initial level, with the exception of Spain and Brazil.

Key words: Positional accuracy, maturity level, uncertainty, accuracy assessment, geometric interoperability.

* Universidad de Jaén, Paraje de las Lagunillas s/n, E-23071, Jaén, España, correo electrónico: fjariza@ujaen.es

** Licencia de Diretoria de Serviço Geográfico Exército Brasileiro, Brasil, correo electrónico: emax0001@red.ujaen.es

*** Sección Nacional del IPGH en Ecuador, Seniergues, 170413, Quito, Ecuador, correo electrónico: elena.chicaiza@ipgh.gob.ec

**** Universidad Politécnica de Madrid, España, correo electrónico: xgoodyear@gmail.com
\end{abstract}




\section{Resumo}

A exatitude posicional constitui um aspecto da qualidade da informação geográfica primordial em qualquer aplicação geomática, e é chave para se lograr interoperabilidade entre conjuntos de dados. O objetivo deste artigo é identificar os métodos de avaliação da qualidade posicional que estão sendo aplicados nos países da Hispanoamérica e, dentro destes, os que participaram do Projeto "Diagnóstico da situação atual sobre as metodologias e procedimentos empregados para a avaliação da qualidade da Informação Geográfica" financiado pelo IPGH. As normas base que são aplicadas correspondem aos métodos americanos como: EMAS, NMAS, NSSDA, PSADG. A maioria destas normas assumem normalidade, mas existem novas tendências como a aberta pela norma espanhola UNE 148002, que supõe uma mudança de paradigma. Finalmente, se apresentam os resultados de um estudo sobre o nível de maturidade nacional com respeito a avaliação da qualidade posicional e que veem a concluir que, nos países analisados, este tipo de avaliações se encontram, na maioria dos casos, em estágio inicial, exceto Espanha e Brasil.

Palavras chave: Precisão de posição, nível de maturidade, incerteza, avaliação de precisão, interoperabilidade geométrica.

\section{Resumen}

La exactitud posicional constituye un aspecto de la calidad de la información geográfica primordial en cualquier aplicación Geomática, y es clave para lograr interoperabilidad entre conjuntos de datos. El objetivo de este artículo es identificar los métodos de la evaluación de la calidad posicional que están siendo aplicados en los países de Hispanoamérica y, dentro de éstos, los que han participado en el Proyecto "Diagnóstico de la situación actual sobre las metodologías y procedimientos empleados para la evaluación de la calidad de la Información Geográfica” financiado por el IPGH. Las normas base que son aplicadas se corresponden con los métodos americanos como: EMAS, NMAS, NSSDA, PSADG. La mayoría de estas normas asumen normalidad, pero hay nuevas tendencias como la abierta por norma española UNE 148002, que supone un cambio de paradigma. Finalmente, se presentan los resultados de un estudio sobre el nivel de madurez nacional con respecto a la evaluación de la calidad posicional y que viene a concluir que, en los países analizados, este tipo de evaluaciones se encuentran, en la mayoría de los casos, en el nivel inicial, exceptuando España y Brasil.

Palabras clave: exactitud posicional, nivel de madurez, incertidumbre, evaluación exactitud, interoperabilidad geométrica. 


\section{Introducción}

La posición es la forma de referir la situación de los objetos en un espacio dado. En este artículo nos referimos a lo que se denomina posicionamiento directo (ISO 19111) o por coordenadas (p.ej. $X=410510, Y=4183127$ ), por oposición al posicionamiento indirecto o por identificadores geográficos (p.ej. Calle Alcalá, $\mathrm{n}^{\circ} 500$, Madrid, España). La exactitud posicional es un aspecto de la calidad de la información geográfica (IG) que resulta primordial en cualquier aplicación geomática. Desde siempre, la componente posicional ha sido considerada como un aspecto definitorio y primordial de la calidad de todo producto cartográfico (Ariza-López, 2002), dado que la posición afecta a la geometría, topología y aspectos temáticos que representan los datos espaciales. Sin embargo, desde hace dos décadas, la componente posicional tiene una renovada importancia en el campo de la Geomática; motivo de ello son, entre otras, las siguientes causas: la difusión de las técnicas y dispositivos GNSS (Global Navigation Satellite Systems, p.ej. el sistema GPS) para la captura de coordenadas, nuevas tecnologías para la toma de datos masivos (p.ej. LIDAR), la implantación de las Infraestructuras de Datos Espaciales (IDE) que requieren interoperabilidad, el incremento de aplicaciones con altas demandas de exactitud posicional (p.ej. inteligencia, militares, vuelos y misiones no tripuladas, agricultura de precisión, etc.), muchas de ellas con carácter global y que requiere posicionamiento absoluto preciso.

A este nuevo marco de grandes exigencias de exactitud posicional se ha venido reaccionando en dos líneas por parte de las agencias cartográficas y productores oficiales de datos:

a) Desarrollando programas de mejora de la exactitud posicional (p.ej. Alemania, Suiza, UK, etc. (Rönsdorf, 2004). La idea es aprovechar los datos levantados por métodos tradicionales que poseen buena calidad en posición relativa en el corto rango, y también gran calidad en cuanto a compleción y aspectos semánticos (p.ej. temáticos), pero que no tienen un nivel suficiente en cuanto a calidad posicional absoluta.

b) Actualización de los métodos de evaluación de la componente posicional ya existentes, o desarrollo de nuevos métodos. Ejemplo en esta línea son el nuevo estándar de la American Society for Photogrammetry and Remote Sensing (ASPRS, 2015), la nueva norma española (UNE 148002) o la nueva norma brasileña (ET-CQDG, 2016).

En la línea de una de las conclusiones del documento "Visión de Aplicación del Marco Normativo de las Américas" (NNUU, 2013), que indica que la normalización geográfica deberá enfocar sus primeros esfuerzos hacia la obtención de un diagnóstico normativo regional, este trabajo se plantea como objetivo el determinar 
la situación actual en lo relativo a los Métodos de Evaluación de la Calidad Posicional (MECP) en los países miembros del Instituto Panamericano de Geografía e Historia (IPGH) que han participado en el Proyecto "Diagnóstico de la situación actual sobre las metodologías y procedimientos empleados para la evaluación de la calidad de la Información Geográfica" <http://coello.ujaen.es/investigacion/ web_giic/SubWeb_IPGH2016/>, financiado en la Convocatoria de Proyectos de Asistencia Técnica del año 2015 (mencionado como Proyecto en adelante).

$\mathrm{El}$ apartado siguiente realiza una aproximación al error posicional $\mathrm{y}$, a partir de la información generada en el Proyecto se identificarán los MECP que se están aplicando, se analizará la situación actual y se valorará el grado de madurez en este aspecto. Finalmente, se incluyen unas conclusiones generales donde se indica la oportunidad de alguna actuación a nivel regional.

\section{Aproximación al Error Posicional, su estimación y control}

Dado un conjunto de datos espaciales (CDE) la posición de un fenómeno del mundo real se describe por medio de valores (coordenadas) en un sistema de referencia. $\mathrm{Su}$ calidad posicional se determina por medio de la exactitud posicional. La exactitud queda definida por ISO 3534-1 como la proximidad o acuerdo entre el resultado de una prueba y el valor aceptado como referencia, de tal manera que se compone de dos términos:

$$
\text { Exactitud }=\text { veracidad }(\text { trueness })+\text { precisión }(\text { precision })
$$

Donde, la veracidad es la proximidad del acuerdo entre el valor promedio obtenido de una serie larga de pruebas y el valor aceptado como referencia; y la precisión es la proximidad del acuerdo entre pruebas independientes obtenidas bajo unas condiciones estipuladas. De forma sencilla podemos indicar que la veracidad es la ausencia de sesgo en la distribución de los errores, y que la precisión es la dispersión que tiene la distribución de los errores y que se relaciona con la variabilidad del proceso aleatorio.

Un aspecto importante en la gestión de los errores posicionales es el modelo que se asume sigue la parte aleatoria (errores aleatorios). Tradicionalmente se considera que éstos se distribuyen según una función de distribución Normal, es decir, según un modelo paramétrico (ecuación matemática que posee unos parámetros). El uso de modelos paramétricos tiene grandes ventajas, pues los datos se sustituyen por los parámetros del modelo y, además, el modelo posee ciertas propiedades que permiten manejarlo convenientemente (p.ej. forma de expandir la incertidumbre a un nivel de confianza) y llegar a conclusiones de manera sencilla (p.ej. por medio de contrastes de hipótesis). En el caso de la distribución Normal los parámetros son la 
media, que permite tomar en cuenta el sesgo (veracidad), y la desviación, que permite modelar la dispersión de los datos (precisión).

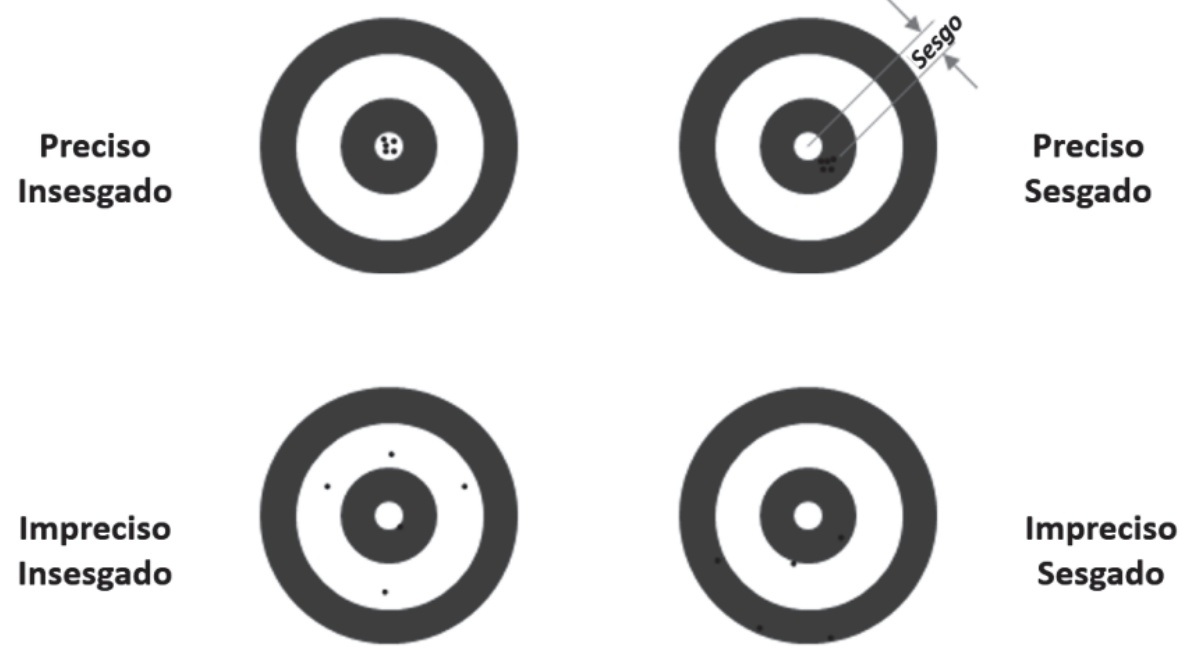

Figura 1. Conceptos de precisión y sesgo.

No obstante, hoy en día está muy en entredicho que los datos de error posicional sean normales, por ejemplo:

- En el caso de datos LIDAR, se indica que pueden ser datos sin distribución alguna (Maune, 2007).

- En la digitalización manual Bolstad y col (1990) indican un modelo bimodal, mientras que Tong y Liu (2004) proponen una p-normal (Normal + Laplace).

- Para el caso de la geocodificación varios autores (Cayo y Talbot, 2003; Karimi y Durcik, 2004; Whitsel y col. 2004) indican la distribución log normal.

- Para observaciones GNSS las distribuciones Raleigh y Weibull (Wilson, 2006; Logsdon, 1995).

- En otros trabajos se mencionan las distribuciones: Normal plegada, HalfNormal, Gamma.

Pensando en métodos estandarizados de evaluación de la componente posicional, básicamente existen dos formas muy distintas de realizar esta evaluación, que son la estimación y el control: 
- Estimación. Significa conocer el valor verdadero de un parámetro que representa una característica de un producto (p.ej. sesgo medio, desviación, etc.), con un cierto nivel de incertidumbre acotado en la estimación. Por tanto, la estimación es la determinación fiable de un valor. En este caso se habla de estimación de la exactitud posicional.

- Control. Significa el deseo de conocer si una característica de un producto alcanza o no un cierto nivel de calidad indicado en unas especificaciones. Por tanto, el control es la determinación fiable de si se cumple o no una condición. En este caso de habla de control de la calidad posicional (control posicional en sentido estricto).

\section{Métodos de evaluación de la calidad posicional identificados}

Nuestro objetivo es identificar los MECP que están siendo aplicados en los países que han participado en el Proyecto y, a partir de aquí, derivar alguna conclusión. Conviene advertir, que no se pretende un análisis técnico en profundidad de estos MECP, pues son ya varios los trabajos que lo han realizado (Ariza-López y Atkinson-Gordo, 2006 y 2008; Nero y Cintra, 2005; Pereira y Nero, 2012), alguno de ellos atendiendo la situación específica en Hispanoamérica (Ariza-López y col., 2007).

La Tabla 1 presenta el conjunto de respuestas que se va a analizar. Atendiendo al orden de las columnas en la tabla, en éstas se indica el país, si éste dispone o no de un MECP publicado como norma nacional, y la referencia a la publicación que lo documenta. En algunos casos, en lugar de la publicación aparece "informante", con ello se quiere indicar que no se existe documentación pública, pero que hay informantes con conocimiento fehaciente de que se está aplicando el método indicado. Conviene advertir que, en algunos casos, los documentos referenciados no son realmente MECP sino especificaciones técnicas que establecen unos criterios que se pueden llevar, de alguna manera, a un MECP.

Se comenzará el análisis por los MECP desarrollados en Estados Unidos por su gran influencia sobre el resto de países; posteriormente se presentará la propuesta del IPGH y, finalmente la de cada uno de los países participantes por orden alfabético.

\section{Estados Unidos}

Son varios los MECP que se han publicado en este país; y muchos de ellos han tenido gran repercusión mundial y que, específicamente, han sido adoptados por países del IPGH. Entre estos métodos están el NMAS (USBB, 1947) y el NSSDA (FGDC, 1998); ambos procedentes de agencias gubernamentales, y varios MECP 
Tabla 1

MECP Identificados

\begin{tabular}{|c|c|c|}
\hline País & Norma & Referencia \\
\hline Argentina (Provincia de Santa Fe) & Sí & $\begin{array}{l}\text { UNL y SCIT (2004). Norma cartográfica de la } \\
\text { Provincia de Santa Fe }\end{array}$ \\
\hline Brasil & $\mathrm{Si}$ & $\begin{array}{l}\text { ET-CQDG (2016): Norma de especificación } \\
\text { técnica para el control de calidad de datos } \\
\text { geoespaciales (ET-CQDG) } \\
\text { Decreto N }{ }^{\circ} 89.817 \text {, del } 20 \text { de junio de } 1984 . \\
\text { Estabelece as Instituciones Reguladoras de } \\
\text { las Normas Técnicas de la Cartografía } \\
\text { Nacional }\end{array}$ \\
\hline Chile & No & $\begin{array}{l}\text { Informantes. Se aplican: NSSDA, PASDG, } \\
\text { EMAS }\end{array}$ \\
\hline Colombia & $\mathrm{Si}$ & $\begin{array}{l}\text { NTC } 5205 \text { - Precisión de datos espaciales } \\
\text { ASPRS (2015). ASPRS Positional Accuracy } \\
\text { Standards for Digital Geospatial Data } \\
\text { IPGH (1978). Especificaciones para mapas } \\
\text { topográficos. Panamá. }\end{array}$ \\
\hline Ecuador & No & Informantes. Se aplica NSSDA. \\
\hline España & $\mathrm{Si}$ & $\begin{array}{l}\text { UNE 148002:2016. Metodología de } \\
\text { evaluación de la exactitud posicional de la } \\
\text { información geográfica }\end{array}$ \\
\hline México & $\mathrm{Si}$ & $\begin{array}{l}\text { INEGI. Norma técnica sobre estándares de } \\
\text { exactitud posicional } \\
\text { INEGI. Compendio de criterios y } \\
\text { especificaciones técnicas para la generación } \\
\text { de datos e informaciones de carácter } \\
\text { fundamental. } 07 \text { Geodesia- } 07.2 \text { Estándares } \\
\text { de Exactitud Posicional }\end{array}$ \\
\hline Panamá & No & Informantes. Se aplican: NSSDA \\
\hline Perú & No & $\begin{array}{l}\text { Instituto Geográfico Nacional, normas } \\
\text { técnicas "especificaciones técnicas para la } \\
\text { producción de mapas topográficos a escala } \\
\text { de } 1: 100,000 \text { " }\end{array}$ \\
\hline Puerto Rico & $\mathrm{Si}$ & $\begin{array}{l}\text { Estándar TIG-005-004: Exactitud posicional } \\
\text { de los datos. Oficina de Gerencia y } \\
\text { Presupuesto }\end{array}$ \\
\hline Uruguay & No & Informantes. Se aplican: NSSDA \\
\hline USA & $\mathrm{Si}$ & $\begin{array}{l}\text { NMAS (USBB, 1947) } \\
\text { NSSDA (FGDC, 1998) } \\
\text { ASPRS (2015) }\end{array}$ \\
\hline Venezuela & No & $\begin{array}{l}\text { IGVSB }(2012) \text {. Especificaciones técnicas para } \\
\text { la revisión de cartografía básica a escala } \\
1: 1,000 \text {. Instituto Geográfico de Venezuela } \\
\text { Simón Bolívar }\end{array}$ \\
\hline
\end{tabular}


procedentes de asociaciones profesionales, de los que cabe destacar el PASDG (ASPRS, 2015) recientemente publicado. Los MECP que se presentan denotan una clara evolución conceptual y mejora a lo largo del tiempo con la intención de adaptarse a las nuevas realidades tecnológicas y necesidades. A continuación, se presentan las ideas básicas de estos métodos:

- NMAS. El United States National Map Accuracy Standards (USBB, 1947) es el método más clásico y difundido en todo el mundo como método de control posicional. Este sencillo método establece dos tolerancias planimétricas $(1 / 30$ " y $1 / 50$ ") en función de dos intervalos de escalas: escalas mayores y escalas menores a 1:20k. A partir de ahí se toma una muestra de puntos de control y se verifica que no más del $10 \%$ de las discrepancias planimétricas sobrepasen la tolerancia aplicable. En altimetría se verifica que no más del 10\% de las elevaciones comprobadas superen un error de más de $1 / 2$ del intervalo entre curvas de nivel. Este método ha recibido notables críticas por su aparente falta de base estadística pero, como demuestran Ariza-López y Rodríguez-Avi (2014), realmente sí posee una base estadística sólida y muy robusta, lo que ocurre es que no se conoce; además, el NMAS ha influido en los MECP de otros países (p.e. IPGH, 1978; Brasil, 1984).

- EMAS. El Engineering Map Accuracy Standards es un método de control posicional desarrollado por la American Society of Civil Engineers (ASCE, 1983), y que se basa en contrastes de hipótesis estadísticas que asumen la normalidad de los datos. Para ello, en cada componente, X, Y y Z, y de manera independiente, se comprueba la existencia o no de un sesgo significativo, y de un comportamiento variacional excesivo. El primer contraste de hipótesis se materializa mediante una prueba $t$ de Student, y el segundo contraste con una prueba Chi2. Es un método muy restrictivo como se demuestra en Ariza-Lopez y col. (2008) debido a que no se corrige la significación total del conjunto de contrastes de hipótesis que se realizan conjuntamente.

- NSSDA. El National Standard for Spatial Data Accuracy es un método de estimación desarrollado por el Federal Geographic Data Committee (FGDC, 1998), que ofrece como resultado un valor de incertidumbre estimado a un nivel de confianza del $95 \%$. De esta forma, es el usuario el que ha de decidir si le resulta adecuado o no a sus propósitos. Para la estimación se toma una muestra reducida de puntos de control (al menos 20 puntos). Se considera la normalidad de los errores planimétricos y, por ello, para la componente planimétrica se asume una distribución Chi2, según los desarrollos de Greenwalt y Shultz (1962). La componente altimétrica se estima de la misma manera y bajo los mismos supuestos, por lo que en este caso se asume la normalidad unidimensional de los errores. 
- PASDG. El Positional Accuracy Standards for Digital Geospatial Data es un método de estimación desarrollado por la American Society of Photogrammetry and Remote Sensing (ASPRS 2015), centrado en la evaluación de ortoimágenes digitales, de datos digitales planimétricos y de datos digitales altimétricos. En este MECP considera clases de exactitud posicional, las cuales se determinan a partir de la estimación de la incertidumbre por medio del cálculo del RMSE sobre una muestra de puntos de control. El PASDG se basa en el NSSDA para los errores que se consideran distribuidos normalmente, y propone el uso de percentiles para el caso de errores altimétricos que se consideren no se distribuyen normalmente. Un avance importante de esta norma es que ya vincula el tamaño de muestra con el tamaño del proyecto fotogramétrico. Un problema de esta norma es que genera dos tipos de resultados, unos para errores normales y otro para errores no normales.

\section{Instituto Panamericano de Geografia e Historia (IPGH)}

Se trata de las Especificaciones Topográficas (IPGH, 1978) que, en lo relativo al control posicional, se alinean en su filosofía con método NMAS (USBB, 1947), que era el estándar más aplicado internacionalmente en esa época. Como diferencia y novedad relevante en su momento, se introdujo un sistema de letras y números que indican categorías de exactitud y requisitos de actualización.

\section{Argentina}

No se ha comprobado la existencia de una norma nacional pero sí se ha identificado la "Norma Cartográfica de la Provincia de Santa Fe" (UNL y SCIT, 2004) realizada por Universidad Nacional del Litoral y Servicio de Catastro e Información Territorial. Se trata de unas especificaciones cartográficas por lo que la parte dedicada al método de evaluación de la componente posicional está realmente ausente. Se basa en el método propuesto por el Decreto Brasileño 89817 de 20 de julio de 1984, si bien reduce los valores que considera para cada tolerancia, haciéndola más estricta.

\section{Brasil}

El Decreto 89817 del 20 de julio de 1984 establece las bases reguladoras de las normas técnicas nacionales y en él se establecen las especificaciones de exactitud posicional a partir del denominado Patrón de Exactitud Cartográfica (PEC). Se distingue entre un PEC planimétrico, para puntos bien definidos, y un PEC altimétrico, para puntos interpolados entre curvas de nivel. El PEC se relaciona linealmente con el Error Patrón $(\mathrm{EP} \approx \mathrm{RMSE})$ de la siguiente manera $\mathrm{PEC}=1.6449 \mathrm{EP}$. El Error Patrón no puede exceder nunca el 60\% del PEC (0.6=1/1.6449). El PEC es un indicador estadístico de la dispersión correspondiente a un nivel de probabilidad 
del $90 \%$, de tal manera que el $90 \%$ de los puntos que se controlen deben tener errores menores que el PEC. Finalmente, se establecen tres clases de exactitud (A, B y $\mathrm{C})$, tal que la clase $\mathrm{C}$ caracteriza productos que poseen un $\mathrm{PEC}$ que es el doble de los productos de clase A. La clase B es una clase intermedia. Vemos en ello que la parte estadística de este método se basa en las ideas del NMAS (USBB, 1947), si bien se establece una condición ( $\mathrm{PEC}=1.6449 \mathrm{EP})$ que denota la adopción explícita del supuesto de normalidad de los errores pues 1.6449 es el $K_{90 \%}$ (caso unidimensional). En los aspectos de clasificación esta norma se basa en la del IPGH (1978). Recientemente ha entrado en vigor la "Norma da especificação técnica para controle de qualidade de dados geoespaciais" (ET-CQDG, 2016). Este documento sí es una verdadera norma centrada en la evaluación de la calidad y, además, es consistente con el marco establecido por ISO 19157 para la calidad de datos espaciales. En cuanto al aspecto espacial se asumen las especificaciones del Decreto 89817 pero se desarrolla todo el marco necesario para el control (definición de los elementos de control, tamaño de muestra, esquema de muestreo, método de informe, etc.). Es de destacar que para la definición de los muestreos se propone la aplicación de la norma ISO 2859 (partes 1 y 2), lo que permite su aplicación a suministros lote a lote y de lotes aislados.

\section{Chile}

No se dispone de documentación oficial que establezca una norma, o en la que adopte una ya existente, pero se tiene conocimiento de la aplicación de los siguientes métodos: NSSDA, EMAS y ASPRS en diversos proyectos cartográficos recientes por parte del Servicio Aerofotogramétrico de la Fuerza Aérea de Chile.

\section{Colombia}

Se dispone de la norma propia NTC 5205 (Instituto Colombiano de Normas Técnicas y Certificación - INCOTEC, 2003), que se aplica conjuntamente con ASPRS (2015) e IPGH (1978). La NTC 5205 se basa en el NSSDA, en cuanto a su base estadística; pero no es una copia literal de ese estándar y en esta línea realiza una argumentación y desarrollo propios, en la que cabe destacar la atención que presta al reporte de los resultados. Respecto a las especificaciones, del Instituto Geográfico Agustín Codazzi (IGAC, 2016) se puede destacar que se incluyen tolerancias para la exactitud posicional relativa.

\section{Ecuador}

Si bien, en los últimos años existe un notable avance en los aspectos de normalización de la IG (Secretaría Nacional de Planificación y Desarrollo — SENPLADES, 
2013), no se dispone de documentación oficial que establezca una norma, o en la que adopte una ya existente.

\section{España}

Recientemente se ha publicado la norma "UNE 148002:2016. Metodología de evaluación de la exactitud posicional de la información geográfica" (Asociación Española de Normalización y Certificación —AENOR, 2016). Esta norma se desvincula del modelo de incertidumbre gaussiano y propone realizar el control posicional a partir de una tolerancia métrica y de la función de distribución de los errores observados. De esta forma se realiza un control basado en las proporciones de error dentro y fuera de tolerancia. Esta sencilla idea permite que su aplicación a los casos 1D, 2D y 3D sin mayor problema (Ariza y Rodríguez, 2014). Conceptualmente, esta norma se puede vincular con el estándar NMAS, si bien supone una clara superación. Para todo lo relacionado con la aceptación la norma UNE 148002 se refiere a la aplicación de la norma internacional ISO 2859 (partes 1 y 2). La norma UNE 148002 está alineada en todo con ISO 19157 y, además, propone un informe de resultados en el que se incluyen elementos de metacalidad, caso único en este estudio.

\section{México}

La "Norma técnica de estándares de exactitud posicional" (Instituto Nacional de Estadística y Geografía - INEGI, 2010b) establece las especificaciones de los órdenes de exactitud posicional. En ella se trabaja con el concepto de círculo de error probable, que se define para un $95 \%$ de intervalo de confianza, y bajo el supuesto de normalidad de los datos (circular para el caso planimétrico y unidimensional para el caso vertical). Según el trabajo de Gómora (2015), se está aplicando el NSSDA con mejoras en cuanto a estratificación de las muestras de control y número de puntos de control, pero todavía no se ha materializado en una norma nacional.

\section{Perú}

En el Instituto Geográfico Nacional (IGN, 2005a y 2005b) se establecen las especificaciones técnicas para la producción de mapas topográficos a escala de 1:100.000 y escala 1:25.000, respectivamente, donde, por la forma de definición de la exactitud posicional, el método de control a aplicar se puede relacionar con el estándar NMAS.

\section{Puerto Rico}

El documento "TIG-005-004: Exactitud posicional de los datos", desarrollado por la Oficina de Gerencia y Presupuesto (OGP, 2011) presenta una traducción del NSSDA, por lo que la adopción de este estándar norteamericano no presenta duda alguna. 


\section{Uruguay}

No se dispone de documentación oficial que establezca una norma o en la que adopte una ya existente, pero se tiene conocimiento de la aplicación del NSSDA en diversos proyectos cartográficos recientes.

\section{Venezuela}

En las especificaciones técnicas para cartografía 1:1,000 (Instituto Geográfico de Venezuela - IGVSB, 2012) se indica el uso del RMSE, estableciéndose una tolerancia planimétrica $(0.15 \mathrm{~m}$ para $1: 1,000)$, pero no se da un criterio explícito de aceptación/rechazo ni un nivel de confianza. Dado que se establece una tolerancia cabe suponer que se trata de un método de control. Es interesante destacar que en este documento no se establece un número mínimo de elementos de control, por el contrario, se indica su máximo $(\mathrm{n}=30)$.

\section{Análisis de la situación}

En este apartado se realiza un doble análisis, el primero centrado en los MECP, es decir, orientado a los estándares que se están aplicando, y el segundo centrado en la madurez que se alcanza, a nivel de país, en lo relativo a la evaluación de la componente posicional.

\section{Situación respecto a los $\mathrm{MECP}$}

Para analizar la situación en cuanto a la evaluación de la componente posicional se parte de los MECP identificados en el apartado anterior. Como ya se ha indicado, existen suficientes trabajos que analizan elementos técnicos de los MECP que se han identificado, por ello este apartado se centrará en analizar la situación regional en relación a la aplicación de MECP.

La Tabla 1 mostraba que se han identificado los MECP aplicados en los países con representantes en este Proyecto (10 casos), y también de otros países sobre los que se ha podido obtener información (tres casos). En esta línea, la Tabla 2 presenta un resumen de lo que denominamos tipologías base, es decir, MECP u otros métodos (p.ej. ISO 2859), que son aplicados directamente, o adaptados, en los países.

Es importante destacar que este conjunto de casos abarca países del norte, del centro y del sur de América, países de gran tamaño (p.ej. Estados Unidos, Brasil, México, Argentina), de tamaño mediano (p.ej. Perú, Venezuela, Colombia) y de tamaño pequeño (p.ej. Ecuador, Uruguay). Este conjunto de casos también recoge diversidad en cuanto al conocimiento y reconocimiento internacional de las instituciones cartográficas generadoras de la cartografía oficial (p.ej. INEGI, IGAC). Por tanto, no se trata de un barrido sistemático ni de un muestreo, pero consideramos que sí es de un conjunto de casos relevante y que puede servir para conocer las alternativas que actualmente se están aplicando y derivar conclusiones. 
Tabla 2

Tipologías base de MECP

\begin{tabular}{|c|c|}
\hline $\begin{array}{c}\text { Clase o } \\
\text { método base }\end{array}$ & Observaciones \\
\hline NMAS & $\begin{array}{l}\text { Estándar procedente de la administración de los Estado Unidos, de } \\
\text { muy simple aplicación y que ha sido ampliamente utilizado en todo el } \\
\text { mundo desde su publicación. Es un método de control que se basa en } \\
\text { contar los elementos de la muestra de control que sobrepasan una } \\
\text { tolerancia. Si la proporción de esos elementos supera un porcentaje } \\
\text { determinado el producto no cumple. En la actualidad se encuentra un } \\
\text { poco denostado por la supuesta falta de base estadística y por estar } \\
\text { diseñado para productos analógicos. En Estados Unidos fue } \\
\text { reemplazado por el NSSDA }\end{array}$ \\
\hline IPGH & $\begin{array}{l}\text { Son las especificaciones cartográficas del Instituto Panamericano de } \\
\text { Geografía e Historia, que fueron adoptadas por numerosos países de } \\
\text { Hispanoamérica en su momento. Se trata de una propuesta de gran } \\
\text { valor para su momento y que se basa en el NMAS pero, a diferencia } \\
\text { con éste, establece varias clases de calidad que conjugan varias } \\
\text { perspectivas (posicional y actualidad) }\end{array}$ \\
\hline EMAS & $\begin{array}{l}\text { Estándar propuesto por la American Society of Civil Engineers. Es un } \\
\text { método de control por medio de varios test de hipótesis estadísticas } \\
\text { basadas en la normalidad de los errores posicionales. Es un método } \\
\text { estricto de control y con gran rechazo, lo que unido a su mayor } \\
\text { complejidad respecto a otros métodos ha supuesto que no tenga tanta } \\
\text { aceptación }\end{array}$ \\
\hline NSSDA & $\begin{array}{l}\text { Estándar procedente del FGDC de Estados Unidos para sustituir el } \\
\text { NMAS. Desde su aprobación en } 1998 \text { ha alcanzado una amplia } \\
\text { difusión y aceptación en todo el mundo. Es un método de estimación } \\
\text { con un tamaño de muestra reducido. Supone la normalidad de los } \\
\text { errores posicionales, la ausencia de sesgo y ciertos límites a la } \\
\text { heterocedasticidad }\end{array}$ \\
\hline PASDG -ASPRS & $\begin{array}{l}\text { Estándar propuesto por la American Society for Photogrammetry and } \\
\text { Remote Sensing, que incorpora el método NSSDA para los cálculos } \\
\text { de estimación sobre datos normales y el uso de percentiles para el } \\
\text { caso de datos no normales. Se establecen clases de exactitud y el } \\
\text { tamaño de muestra se relaciona con el tamaño del proyecto } \\
\text { fotogramétrico }\end{array}$ \\
\hline ISO 2859 & $\begin{array}{l}\text { Norma ISO internacional que se aplica ampliamente en la industria. } \\
\text { Sirve para el control de características del tipo atributo en suministros } \\
\text { lote a lote y en lotes aislados. No requiere normalidad en los datos. Se } \\
\text { basa en el conteo de elementos defectuosos dentro de una muestra. En } \\
\text { este método se evidencian los riesgos del productor y del adquiriente. } \\
\text { Utilizan el nivel de calidad aceptable (NCA) como parámetro para la } \\
\text { indexación con la calidad, por lo que se requiere cierta adaptación al } \\
\text { caso de los errores métricos }\end{array}$ \\
\hline
\end{tabular}


Ya se ha advertido con antelación que, en algunos casos, los documentos referenciados no son realmente $\mathrm{MECP}$ sino especificaciones técnicas que establecen unos criterios que se pueden llevar, de alguna manera, a un MECP. Esta situación ocurre en cuatro casos. Dejando a un lado Estados Unidos, España y Puerto Rico, que como Estado asociado a Estados Unidos ha de cumplir sus exigencias legales, sólo Colombia y Brasil poseen un documento normativo centrado en los aspectos de valuación. Además de los ya indicados, hay cuatro casos en lo que no existe ningún tipo de documentación pública oficial.

En cuanto a disponer o no de una norma nacional publicada, sólo tres países están en esta situación (Brasil, Colombia y México), a los que se pueden sumar, Puerto Rico, España y el propio Estados Unidos. No existe ningún país que tenga una referencia explícita a la adopción de un MECP de terceros (p.ej. adopción del NSSDA). Por lo tanto, son mayoría amplia (7) los países que no disponen de una norma nacional publicada, con independencia de que puedan existir normas en provincias o estados, como es el caso de Argentina. Indudablemente esto no significa que en estos últimos países no se estén realizando evaluaciones de la componente posicional, pero lo que sí refleja es una situación de cierta "informalidad", que genera falta de transparencia y, por ende, desconfianza.

Otro aspecto relevante es la fecha de los MECP. Según se observa, los últimos años han sido de gran actividad en este campo, pues entre 2015 y 2016 se han publicado normas en Estados Unidos (ASPRS, 2015), España (UNE 148002:2016) y Brasil (ET-CQDG, 2016), lo que indica el interés sobre esta temática. Además, estas tres normas presentan notables innovaciones respecto al panorama anterior, y una evolución que se preocupa y ocupa con más rigor de los aspectos estadísticos y formales.

Otro elemento que interesa destacar es la evolución/adaptación a lo largo del tiempo de los MECP dentro de un mismo país. El caso más evidente es Estados Unidos, que con los numerosos estándares publicados (NMAS, EMAS, ASPRS, NSSDA, PASDG-ASPRS) muestra una clara secuencia de MECP que van evolucionando para adaptarse a las tecnologías y exigencias de cada momento. Una evolución así evidencia una línea clara de preocupación y mejora sostenida a lo largo del tiempo en lo relacionado con la evaluación de la componente posicional. Del resto de países destaca Brasil que con la norma ET-CQDG-2016 perfecciona y mejora lo indicado hace más de 30 años en el Decreto No 89.817 (20 de junio de 1984).

Entrando en aspectos relacionados con la filosofía de los MECP, un análisis somero de los casos presentados en el apartado anterior permite identificar fácilmente el método sobre el que se basa cada uno de ellos, y concluir que la mayoría de los MECP identificados se alinean con las propuestas procedentes de Estados Unidos o el IPGH. La Tabla 3 presenta estos alineamientos. 
Tabla 3

Tipologías base de MECP por país

\begin{tabular}{|c|c|c|c|c|c|c|}
\hline \multirow[t]{2}{*}{ País } & \multicolumn{6}{|c|}{ Norma/documento al que se alinea } \\
\hline & $N M A S$ & $I P G H$ & $E M A S$ & NSSDA & $A S P R S$ & ISO2859 \\
\hline Argentina (Santa Fe) & & $\mathrm{X}$ & & & & \\
\hline Brasil & $\mathrm{X}$ & $\mathrm{X}$ & & & & $\mathrm{X}(1)$ \\
\hline Chile (SAF) & & & $\mathrm{X}$ & $\mathrm{X}$ & $\mathrm{X}$ & \\
\hline Colombia & & & & $\mathrm{X}$ & $\mathrm{X}$ & \\
\hline Ecuador & & & & $\mathrm{X}$ & & \\
\hline España & & & & & & $\mathrm{X}(1)$ \\
\hline IPGH & $\mathrm{X}$ & & & & & \\
\hline México & & & & $\mathrm{X}$ & & \\
\hline Panamá & & & & $\mathrm{X}$ & & \\
\hline Perú & $\mathrm{X}$ & & & & & \\
\hline Puerto Rico & & & & $\mathrm{X}$ & & \\
\hline Uruguay & & & & $\mathrm{X}$ & & \\
\hline USA & & & $\mathrm{X}$ & $\mathrm{X}$ & $\mathrm{X}$ & \\
\hline Venezuela & $X(3)$ & & & & & \\
\hline
\end{tabular}

(1) Se utiliza para determinar tamaños de muestra y criterios de aceptación y rechazo conjugados con un criterio(s) de toleracias métricas.

(3) La interpretación de la tolerancia correspondiente a un nivel de confianza permite asemejar este método al NMAS.

En este panorama, dejando a un lado Estados Unidos y España, destaca la gran influencia de los métodos NMAS y NSSDA procedentes de USA. Teniendo en cuenta que la norma del IPGH se basa en el NMAS, este y el NSSDA se reparten, casi por igual, la totalidad de los casos. Lo que significa básicamente el uso equilibrado de las dos formas ya indicadas de realizar la evaluación de la componente posicional, que son la estimación y el control. Nos puede quedar la duda de si es una elección consciente o una situación heredada de la evolución temporal de las normas en Estados Unidos y su adopción desigual por el resto de países.

También es destacable que los casos de Chile y Colombia se han indicado varios MECP. Esto es interesante dado que puede indicar una utilización selectiva de cada uno de ellos en función de las posibles problemáticas en la evaluación posicional; sin embargo, también puede ser un problema a la hora de interoperar con los resultados. Otro aspecto en el que destacan estos dos países es que ambos están ya apli- 
cando el método ASPRS de muy reciente publicación, lo que indica una gran capacidad de adopción de nuevos métodos.

En otro orden, por su ruptura con la homogeneidad general, se han de considerar los casos de España (norma UNE 148002) y de Brasil (norma ET-CQDG), ambas de muy reciente aprobación. En ambos casos se establece el uso de la Norma Internacional ISO 2859. La Norma Internacional ISO 2859 (partes 1 y 2) se dedica al establecimiento de planes de muestreo para la aceptación de productos que se controlan por medio de atributos, en el primer caso (ISO 2859-1) para suministros lote a lote y, en el segundo (ISO 2859-2), para suministros de lotes aislados. Esta nueva dirección supone aspectos interesantes como, la alineación con estándares del ámbito industrial, poder utilizar para la posición índices de calidad (nivel de calidad aceptable) comunes a otros aspectos de la calidad (p.ej. compleción, tema, consistencia, etc.), y desvincularse de las exigencias de normalidad sobre los errores posicionales.

En los trabajos ya referidos de Ariza-López y Atkinson-Gordo (2006, 2007 y 2008), Nero y Cintra (2005) y Pereira y Nero (2012) se puede encontrar un análisis técnico de los MECP anteriores al año 2012, por lo que estos análisis siguen siendo válidos para la mayoría de los métodos identificados en este trabajo (p.ej. NMAS, EMAS, NSSDA). A modo de resumen, la Tabla 4 presenta las principales debilidades y aspectos mejorables en los MECP al uso. Estas son debilidades que normalmente son heredadas por quienes adoptan o adaptan los mencionados MECP. Las tres normas más recientes (PASDG-ASPRS, UNE 148002 y ET-CQDG) sí muestran una clara mejora en relación a algunos de los aspectos que se indican en la Tabla 5, existiendo todavía mucho recorrido para posibles mejoras. Algunos de los aspectos presentados en la Tabla 5 serán tomados en cuenta a la hora de estudiar la madurez alcanzada (apartado siguiente).

\section{Madurez país en lo relativo a la evaluación de la componente posicional}

En la vida cotidiana entendemos por madurez el grado de desarrollo de algún aspecto, por ejemplo, en las personas la madurez psíquica, la madurez emocional o la madurez física. En este caso entendemos madurez en la evaluación de la componente posicional como el grado de desarrollo de que se ha alcanzado en estos procesos, con un sentido amplio y relacionado con la capacidad de mejora continua. El uso de modelos de madurez es una técnica usual en muchas ramas, entre ellas la empresarial, el desarrollo del software, la prestación de servicios, etc., $<$ https://en.wikipedia.org/wiki/Maturity_model $>$. Básicamente un modelo consiste en establecer un conjunto de niveles de madurez (p.ej. nulo, inicial, medio, avanzado, etc.), y un conjunto de medios (p.ej. encuestas, evidencias, etc.) para asignar un nivel a cada elemento que se evalúa. 
Tabla 4

Aspectos mejorables en los MECP

\begin{tabular}{|c|c|}
\hline Aspectos formales & $\begin{array}{l}\text { Los documentos que establecen los MECP no siempre siguen } \\
\text { la estructura convencional y estilo de redacción que } \\
\text { corresponde a una norma redactada por un cuerpo normativo }\end{array}$ \\
\hline Control aislado & $\begin{array}{l}\text { La mayoría de los MECP sólo consideran evaluaciones de } \\
\text { conjuntos de datos aislados, por lo que se pierde la capacidad } \\
\text { de un control más eficiente y eficaz que se podrían aplicar a } \\
\text { flujos de suministros }\end{array}$ \\
\hline Requisitos & $\begin{array}{l}\text { No se orienta sobre requisitos de exactitud, o sólo se } \\
\text { relacionan con la escala. En pocos casos se vincula con la } \\
\text { situación actual en las aplicaciones geomáticas (p.ej. } \\
\text { agricultura de precisión) }\end{array}$ \\
\hline Población a controlar & $\begin{array}{l}\text { La determinación de la población de elementos que se controla } \\
\text { no siempre está explícita, ni se insiste en su identificación }\end{array}$ \\
\hline Elemento de control & $\begin{array}{l}\text { La mayoría de los MECP se basan exclusivamente en puntos } \\
\text { bien definidos y fácilmente identificados como elementos de } \\
\text { control, y no son válidos para otras tipologías (p.ej. líneas) }\end{array}$ \\
\hline Tamaño de muestra & $\begin{array}{l}\text { En los métodos de estimación no siempre está relacionado con } \\
\text { el tamaño de la población, y se proponen tamaños de muestra } \\
\text { poco adecuados para la estimación (p.ej. al menos } 20 \text { puntos } \\
\text { de control) }\end{array}$ \\
\hline Distribución espacial & $\begin{array}{l}\text { No suele darse directrices sobre la distribución espacial de la } \\
\text { muestra de control }\end{array}$ \\
\hline Exactitud del control & $\begin{array}{l}\text { Se suele indicar la necesidad de una mayor exactitud en los } \\
\text { trabajos de control, pero normalmente no se ofrecen } \\
\text { coeficientes de seguridad para hacer ajustes, ni métodos de } \\
\text { cálculo cuando no se puede asegurar esa mayor exactitud }\end{array}$ \\
\hline Exactitud relativa & No suele incluirse en el MECP \\
\hline $\begin{array}{l}\text { Hipótesis estadísticas } \\
\text { subyacentes }\end{array}$ & $\begin{array}{l}\text { No siempre se explicitan (p.ej. normalidad, } \\
\text { homocedasticidad). Por lo general no se indica que se deban } \\
\text { comprobar ni el método de comprobación }\end{array}$ \\
\hline Atípicos & $\begin{array}{l}\text { No se indica su relevancia ni cómo tratarlos, ni la necesidad de } \\
\text { incluirlos en los informes }\end{array}$ \\
\hline Nivel de confianza & No existe acuerdo sobre el nivel a considerar (p.ej. 90\% o 95\%) \\
\hline Normalidad & $\begin{array}{l}\text { La mayoría de los MECP se basan en suponer la normalidad } \\
\text { de los errores posicionales por lo que no son adecuados } \\
\text { cuando no esta hipótesis no es cierta (p.ej. control de } \\
\text { elementos lineales por orlados, control de MDE en áreas } \\
\text { vegetadas, etc.) }\end{array}$ \\
\hline Información y guías & $\begin{array}{l}\text { Los MECP no suelen incluir una información suficiente, } \\
\text { detallada, con ejemplos y comentarios que facilite su } \\
\text { aplicación }\end{array}$ \\
\hline ISO 19157 & $\begin{array}{l}\text { La mayoría de los casos no usa las medidas de calidad } \\
\text { propuestas por esta norma, o por su predecesora (ISO 19138) }\end{array}$ \\
\hline Metacalidad & $\begin{array}{l}\text { No suele existir indicación de la propia incertidumbre del } \\
\text { método de control }\end{array}$ \\
\hline Riesgos & $\begin{array}{l}\text { Los riesgos del productor (error tipo I) y del usuario (error tipo } \\
\text { II) no siempre están explícitos, ni se mencionan }\end{array}$ \\
\hline
\end{tabular}


Dentro del equipo de trabajo del Proyecto se ha desarrollado un modelo de madurez-país relativo a la calidad de la información geográfica usando los conceptos de Modelo de Madurez de Capacidades (Paulk et al., 1993), del que aquí se presenta la parte del modelo que está relacionada con la calidad posicional. Para este modelo se han considerado los niveles que se presentan en la Tabla 5. Se parte de un nivel nulo en el que, o bien no existen evaluaciones de la calidad posicional, o son muy escasas. Considerando que la exactitud posicional es el aspecto más clásico de la calidad de los datos espaciales, el nivel medio (M) es el que se considera debería ser el común para la mayoría de los países de un entorno desarrollado. Los niveles avanzado (A) y óptimo $(\mathrm{O})$ plantean los retos que se han de satisfacer en un futuro cercano dentro de esta componente de la calidad.

Tabla 5

Grado de madurez en MECP

\begin{tabular}{|c|c|}
\hline Nivel & Definición \\
\hline Nulo & No se realizan evaluaciones de la calidad posicional, o son muy escasas \\
\hline Inicial (I) & $\begin{array}{l}\text { Sólo se realizan evaluaciones de la calidad posicional en algunos } \\
\text { productos y sólo por parte de las agencias oficiales }\end{array}$ \\
\hline Medio (M) & $\begin{array}{l}\text { Todos los productores realizan evaluaciones de la calidad posicional de } \\
\text { todos sus productos según normas propias o de terceros. Informando en } \\
\text { metadatos de tipo estándar. Dichas evaluaciones son aún sobre ámbitos } \\
\text { generales }\end{array}$ \\
\hline Avanzado (A) & $\begin{array}{l}\text { Se evalúa la calidad posicional por tipologías de elementos utilizando } \\
\text { técnicas de evaluación de puntos para puntos, de líneas para líneas y de } \\
\text { superficies para superficies. Se informa de manera independiente para } \\
\text { cada tipología y temática }\end{array}$ \\
\hline Óptimo (O) & $\begin{array}{l}\text { La posición está fuertemente vinculada con modelos de error espacial y } \\
\text { mecanismos de evaluación automatizados, y el modelo se revisa de } \\
\text { manera continua }\end{array}$ \\
\hline
\end{tabular}

Para poder asignar niveles se han de desplegar un conjunto de cuestiones que permitan, de una forma objetiva, asignar unas valoraciones según su contestación. Estas preguntas suelen estructurarse según áreas estratégicas, tácticas y operativas, pueden ser de cualquier tipología y en el número que se considere adecuado. Las cuestiones podrán contestarse preguntando a personas (p.ej. expertos, usuarios, etc.) o pueden basarse en evidencias (p.ej. número de normas, número de accesos a un servicio(s), etc.).

En el caso que nos ocupa se han considerado las áreas táctica y operativa, y las cuestiones, o prácticas, que se presentan en la Tabla 6 para cada uno de los niveles (I, M, A, O). A cada una de estas cuestiones o prácticas se les asigna una ponderación que refleje la importancia relativa que poseen. 
Tabla 6

Ponderación del grado de madurez en MECP

\begin{tabular}{|c|c|c|c|}
\hline $\begin{array}{c}\text { Área- } \\
\text { Categoria }\end{array}$ & Nivel & Prácticas & Ponderación \\
\hline \multirow{7}{*}{ 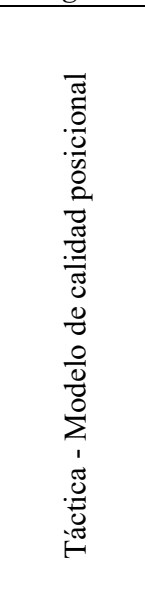 } & I & Existe un modelo de calidad posicional definido & 1 \\
\hline & M & $\begin{array}{l}\text { El modelo de calidad posicional prevé informes en } \\
\text { metadatos de tipo estándar }\end{array}$ & 1 \\
\hline & A & $\begin{array}{l}\text { El modelo de calidad posicional permite evaluación } \\
\text { por tipologías de elementos }\end{array}$ & 0.4 \\
\hline & & $\begin{array}{l}\text { El modelo de calidad posicional describe técnicas de } \\
\text { evaluación de puntos para puntos, de líneas para } \\
\text { líneas y de superficies para superficies }\end{array}$ & 0.3 \\
\hline & \multirow{3}{*}{$\mathrm{O}$} & $\begin{array}{l}\text { El modelo de calidad posicional prevé informes en } \\
\text { ámbitos distintos para tipología y/o temática de los } \\
\text { elementos }\end{array}$ & 0.3 \\
\hline & & $\begin{array}{l}\text { El modelo de calidad posicional está fuertemente } \\
\text { vinculado con modelos de error espacial }\end{array}$ & 0.4 \\
\hline & & $\begin{array}{l}\text { El modelo de calidad posicional se revisa de manera } \\
\text { continua con una frecuencia predeterminada }\end{array}$ & 0.6 \\
\hline \multirow{6}{*}{ 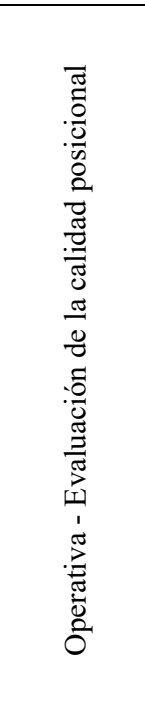 } & I & $\begin{array}{l}\text { Es posible identificar en los metadatos de la IDE } \\
\text { nacional más de un producto con informe sobre } \\
\text { evaluación de calidad posicional }\end{array}$ & 1 \\
\hline & M & $\begin{array}{l}\text { La mayoría }(>60 \%) \text { de los productores públicos de } \\
\text { IG tiene algún registro de evaluación de calidad } \\
\text { posicional en la IDE nacional }\end{array}$ & 0.7 \\
\hline & & $\begin{array}{l}\text { Más de } 5 \% \text { de los productos en la IDE nacional } \\
\text { cuenta con datos de la evaluación de su calidad } \\
\text { posicional, aunque en un ámbito general }\end{array}$ & 0.3 \\
\hline & A & $\begin{array}{l}\text { Todos los productos en la IDE nacional con } \\
\text { evaluación de calidad posicional siguen el modelo de } \\
\text { calidad válido en su fecha de creación }\end{array}$ & 0.5 \\
\hline & & $\begin{array}{l}\text { Hay registros de evaluación de calidad posicional por } \\
\text { tipologías de elementos en productos que cuentan } \\
\text { con más de una tipología }\end{array}$ & 0.5 \\
\hline & $\mathrm{O}$ & $\begin{array}{l}\text { Existen mecanismos de evaluación automática de la } \\
\text { calidad posicional disponibles a los usuarios de IG en } \\
\text { la IDE nacional }\end{array}$ & 1 \\
\hline
\end{tabular}

Debe entenderse que un modelo debe ser útil a lo largo de mucho tiempo, y por ello debe ser dinámico y desarrollarse con la propia evolución de los conocimientos y capacidades tecnológicas de cada momento. Por ello, tanto el alcance de los niveles de madurez, como la consideración de los aspectos estratégicos, tácticos y operativos, como las prácticas o cuestiones y las ponderaciones podrán ser cambiadas a 
lo largo del tiempo para que el modelo siga siendo realmente útil. Según lo dicho, un modelo siempre debe incorporar su fecha y versión.

El modelo presentado anteriormente ha sido desarrollado en el seno de los participantes del Proyecto. El Modelo de Madurez fue debatido entre sus realizadores y el resto de participantes del Proyecto durante el taller que tuvo lugar la sede del IPGH en Quito del 15 al 17 de noviembre de 2016. Finalmente, los representantes de cada país valoraron la situación de su país según el modelo. Los resultados de esta autovaloración indican que, todos los países que participaron del proyecto se encuentran en un nivel inicial, hecha la excepción de Brasil y España, quienes están cerca del nivel medio.

\section{Conclusiones}

La calidad posicional es una componente primordial de la calidad de los datos espaciales, y un elemento básico para conseguir la interoperabilidad de productos y servicios geoespaciales. La calidad posicional se evalúa por medio de los MECP que se pueden clasificar en estimatorios (obtienen un valor representativo) y de control (aceptan o rechazan en función de una especificación). En este trabajo se ha analizado la situación de los MECP en 10 países de Hispanoamérica dentro del Proyecto "Diagnóstico de la situación actual sobre las metodologías y procedimientos empleados para la evaluación de la calidad de la Información Geográfica".

Como conclusión principal se puede indicar que en la mayoría de los casos analizados se tiene como base de la evaluación posicional métodos procedentes de Estados Unidos. Esto métodos son el NMAS y el NSSDA. En algunos casos estas normas se aplican de manera directa, si bien en otros casos han sido adaptadas en su redacción buscando una mejor adecuación a la realidad particular. La norma del IPGH (1978) también se menciona en algunos países. De modo general, se aprecia un claro interés por mantenerse actualizados y mejorar los procesos de evaluación de la componente posicional, lo que lleva a adoptar los cambios que van surgiendo fuera de sus fronteras. El principal problema que se detecta es el que se ha denominado "informalidad", es decir, la situación en la que no se dispone de una norma nacional publicada o una referencia explícita a la adopción de un método concreto (p.ej. IPGH, NMAS, NSSDA, etc.). Esta situación se da en una mayoría de casos (siete países) y bajo nuestro punto de vista genera falta de transparencia y, por ende, desconfianza.

Dentro del grupo analizado se detecta la existencia de notables diferencias. Algunos países sólo disponen de unas especificaciones muy generales que pudieran ser tomadas como base de los MECP, y otros disponen de normas bien desarrolladas. Dentro del grupo analizado destacan los casos de España (norma UNE 148002) y de Brasil (norma ET-CQDG), los dos de muy reciente aprobación. En ambos casos se utiliza la Norma Internacional ISO 2859 como base del método de acepta- 
ción. Esta nueva manera supone aspectos interesantes como la alineación con estándares del ámbito industrial, poder utilizar para la posición índices de calidad (nivel de calidad aceptable) comunes a otros aspectos de la misma (p.ej. compleción, tema, consistencia, etc.), y desvincularse de las exigencias de normalidad sobre los errores posicionales.

Apoyados en trabajos previos, se han indicado las principales debilidades y aspectos mejorables en los MECP. Estos aspectos son numerosos y variados, y cubren aspectos de forma, de base estadística, de aplicabilidad, de información para el buen uso, etc. Las normas más recientes (p.ej. PASDG, UNE 148002 y ETCQDG) ya incluyen mejoras parciales en numerosos aspectos.

Dentro de este trabajo también se ha realizado una valoración del grado de madurez el campo de la evaluación de la calidad posicional de los datos espaciales. Se han definido unos niveles de madurez para las áreas táctica y operativa con sus respetivas categorías modelo de calidad posicional y evaluación de calidad posicional, con esta ponderación se ha realizado la autovaloración por parte de los participantes en el Proyecto. Resultado de esta parte del modelo es que el grado de avance en los aspectos relativos los MECP los países en su mayoría se encuentran en el nivel inicial, exceptuando España y Brasil.

En resumen, el análisis realizado nos permite estimar que estamos ante una situación en la que, consideramos que cualquier actividad de liderazgo por parte del IPGH en este campo, tiene potencialidad de ser bien acogida por muchos países de la región. En este sentido, nos permitimos recoger aquí una de las principales conclusiones del informe "Visión de Aplicación del Marco Normativo de las Américas" (NNUU, 2013):

Es preciso definir y adoptar un marco normativo compatible en la región, que establezca las convenciones comunes y acuerdos técnicos necesarios para alcanzar mayor eficiencia en la respuesta a las demandas de información geoespacial, donde los datos se generen y mantengan el común denominador de: compatibilidad, comparabilidad, compartibilidad, confiabilidad, consistencia y completez, siendo éste la base para el establecimiento de un esquema interoperable de colaboración, que contribuya al desarrollo de IDEA.

De una manera más concreta y específica, consideramos que el IPGH debería seguir apoyando acciones de capacitación y normalización comunes a todos los países americanos en el ámbito de la calidad de los datos espaciales y, particularmente por su importancia, en el de la calidad posicional. En esta última línea, la Tabla 4 y el Modelo de Madurez (Tabla 6) presentan numerosos aspectos que convendría mejorar, así como directrices de mejora. 


\section{Agradecimientos}

Al IPGH por la financiación del Proyecto "Diagnóstico de la situación actual sobre las metodologías y procedimientos empleados para la evaluación de la calidad de la Información Geográfica" de la convocatoria de Asistencia Técnica de 2016, financiado por las comisiones de Cartografía y Geografía.

A los miembros del proyecto e informantes: Ricardo Mansilla de Argentina, a Emerson Xavier de Brasil, a Xavier Buenaño de Ecuador, a Pablo Morales de Chile, a Ana Morales de Colombia, a Héctor Gómora de México, a Elisenia Mendoza de Panamá, a Edison Rosas de Uruguay y a Elizabeth Vargas de Venezuela.

\section{Bibliografía}

AENOR (2016). UNE 148002:2016. Metodología de evaluación de la exactitud posicional de la información geográfica.

Ariza, F.J.; Atkinson, A.D.J. (2006). Metodologías de control posicional: visión general y análisis crítico. Informe al CT-148 de AENOR. GIIC, Universidad de Jaén.

Ariza-López, F.J.; Atkinson-Gordo, A.J. (2008). “Analysis of some positional accuracy assessment methodologies", Journal of surveying Engineering.

ASCE (1983). Map Uses, scales and accuracies for engineering and associated purposes, American Society of Civil Engineers, Committee on Cartographic Surveying, Surveying and Mapping Division, New York.

ASPRS (1990). “Accuracy standards for large scale maps", PE\&RS, 56(7):10681070.

- (2015). "ASPRS Positional accuracy standards for digital geospatial data", Photogrammetric Engineering \& Remote Sensing, 81(3):53, $<$ http://www.asprs.org/Standards-Activities.html $>$.

Brasil (1984). Decreto $n^{\circ} 89817$ de 20/07/1984 por el que se establecen las Instrucciones reguladoras de las normas técnicas de cartografía nacional, Brasilia.

Colombia (2006). Parte 3, Estándar nacional para la precisión de los datos espaciales (EPDE), Comité Técnico Incotec 34, $<$ http://codazzi4.igac.gov.co/comite034/documentos/posicionamiento/parte3.pdf $>$, accedida el 29 de octubre de 2006.

Ecuador (2006). Especificaciones técnicas generales para la realización de cartografia topográfica a cualquier escala (primer borrador: IGMC-PG-N-ET-01), disponible en <http://www.igm.gov.ec/ downloads/especcarto3.html>.

FGDC (1998). FGDC-STD-007: Geospatial Positioning Accuracy Standards, Part 3. National Standard for Spatial Data Accuracy, Federal Geographic Data Committee, Reston. 
(2003). Revision of Geospatial Positioning Accuracy Standards, Part 3. National Standard for Spatial Data Accuracy, FGDC-STD-007.3-1998, Federal Geographic Data Committee, < http://www.fgdc.gov/standards/projects/FGDCstandards-projects/accuracy/part3/index_html>, September 20, 2006.

Gómora, H.M. (2015). "La calidad como aptitud para el uso: evaluación de la exactitud posicional de los productos topográficos digitales a escala 1:50.000 del INEGI", en Revista Cartográfica, 91:159-174.

ICONTEC (2000). Conceptos Básicos de Calidad DE631/00. Disponible en $<$ http://codazzi4.igac.gov.co/comite034/documentos/consulta_publica/DE631-00_ Calidad.pdf $>$.

(2002). NTC5043-Conceptos básicos de calidad de los datos geográficos, Santa Fé de Bogotá (Colombia), también disponible en Colombia (2006).

IGAC (1994). Resolución 64 de 07 de enero de 1994: "Umbrales de exactitud y precisión en posición”, Instituto Geográfico Agustín Codazzi, Bogotá, Colombia.

(2016). Especificaciones técnicas cartografía básica digital.

IGN (2005a). Especificaciones técnicas para la producción de mapas topográficos a escala de 1:100,000, Instituto Geográfico Nacional del Perú.

(2005b). Especificaciones técnicas para la producción de mapas topográficos a escala de 1:25,000.

IGVSB (2012). Especificaciones técnicas para la revisión de cartografía básica a escala 1:1,000. Instituto Geográfico de Venezuela Simón Bolívar.

INCOTEC (2003). NTC 5205: Norma Técnica Colombiana. Precisión de datos espaciales.

INEGI (2010a). Compendio de criterios y especificaciones técnicas para generación de datos e información de carácter fundamental. 07 Geodesia. 07.2. Estándares de exactitud posicional.

(2010b). Norma técnica de estándares de exactitud posicional.

IPGH (1978). Especificaciones para mapas topográficos, Panamá, Instituto Panamericano de Geografía e Historia.

ISO (1985). ISO 2859-2: Sampling procedures for inspection by attributes - Part 2: Sampling plans indexed by limiting quality (LQ) for isolated lot inspection. International Organization for Standardization, Geneva.

(1993). International vocabulary of basic and general terms in metrology, International Organization for Standardization, Geneva.

- (1995). Guide to the expression of uncertainty in measurements, International Organization for Standardization, Geneva.

(1999). ISO 2859-1: Sampling procedures for inspection by attributes Part 1: Sampling schemes indexed by acceptance quality limit (AQL) for lot-bylot inspection, International Organization for Standardization, Geneva. 
México (2005). Propuesta preliminar de diseño de prueba para la evaluación de la exactitud posicional de productos cartográficos a escalas mayores, aplicando la metodología del "Minnesota Department of Transportation".

Nero, M. (2006). Metodologias avançadas no controle de qualidade posicional de bases cartográficas, Relatório de Pós-doutorado Júnior, Escola Politécnica da USP, São Paulo.

Nero, M. y Cintra, J. (2005). “Control de calidad de la cartografía: visión general de las normas de diversos países”, en XXII Congresso Brasileiro de Cartografia, Macaé-RJ, SBC.

(2005). Controle de qualidade de mapeamiento: Visao general das normas de diversos países, Departamento de Engenharia de Transportes, Escola Politécnica da USP.

Nero, M.A. (2005). "Propuestas para el control de calidad de bases cartográficas con énfasis en la componente posicional", tesis doctoral, Escuela Politécnica de la USP, Sao Paulo.

NNUU (2013). Visión de Aplicación del Marco Normativo de las Américas CPIDEA, disponible en <http://unstats.un.org/unsd/geoinfo/RCC/docs/rcca10/E\% 20Conf_103_15_CPIDEA_MarcoNormativo_ESP_final.pdf $>$.

Paulk, M.C.; Curtis, N.; Chrissis, M.B. and Weber C.V. (1993). Capability Maturity Model for Software, Version 1.1. Technical Report CMU/SEI-93-TR-024 ESCTR-93-177, disponible en <https://www.sei.cmu.edu/reports/93tr024.pdf>.

Pereira, T.A.J.; Nero M.A. (2012). “Análise de normas de controle de qualidade posicional em cartografia ao redor do mundo: exemplos atuais de alguns países", IV Simpósio Brasileiro de Ciências Geodésicas e Tecnologias da Geoinformação, Recife, PE, 06-09 de maio, p. 1-9.

Rönsdorf, C. (2004). Positional integration of geodata, EuroSDR, disponible en $<$ http://bono.hostireland.com/ eurosdr/km_pub/no48/workshops_docs/positiona 1_accuracy_dit_march_05/Positional_Integration_of_Geodata_Carsten_Roensdo rf.pdf $>$.

SENPLADES (2013). Estándares de Información Geográfica, disponible en $<\mathrm{http:}$ //app.sni.gob.ec/sni-link/sni/PORTAL_SNI/PORTAL/CONAGE/T/14_ estandares_de_informacion_geografica.pdf $>$.

UNL y SCIT (2004). Norma Cartográfica de la provincia de Santa Fe, Universidad Nacional del Litoral y Servicio de Catastro e Información Territorial.

USBB (1947). United States National Map Accuracy Standards, U.S. Bureau of the Budget, Washington. 